\title{
Research on the Securitization of
}

\section{Payment Guarantees of the Micro-credit Company}

\author{
Xin Sui* \\ School of Finance \\ Nanjing University of Finance and Economics \\ Nanjing, P.R.China
}

\author{
Zhenghong Tang \\ Jiangsu Re-guarantee Group \\ Nanjing, P.R.China
}

\begin{abstract}
The development of the business of payment guarantees of the micro-credit company broadens the sources of funds. While the implementation of payment guarantee securitization will further broaden the financing channels of the micro-credit company. This improves its financing dilemma. During the process of the payment guarantee securitization, the pricing plays an important role. Based on this point, the necessity of payment guarantee securitization, the construction of the asset pool and the flow path of securitization, and the pricing of the securitization of payment guarantees of the micro-credit company are respectively researched in this paper.
\end{abstract}

Keywords-micro-credit company; payment guarantee; securitization of guarantee

\section{INTRODUCTION}

The birth of the micro-credit company is one of the breakthroughs in the reform of Chinese monetary system, and it is a new emerging financial form developed for the purpose of perfecting inclusive financial system and meeting the strong demand of economic development [1]. Its establishment and development do provide more convenient loans and other financial services for the low income groups and small and micro businesses, and can improve the efficiency of private capital [2]. However, during the process of its development, financing plight emerges [1] [3]. It is necessary to broaden the financing channels of the micro-credit company. The products of the right of return on assets of the micro-credit company were first traded in Chongqing financial assets exchange on July 19, 2011[4]. There is no doubt that it creates a new financing channel for the micro-credit company and also results in a new era of financial innovation for the micro-credit company [5]. It is of great significance for the micro-credit company to carry out the securitization of payment guarantees in such an environment. The micro-credit company has a large amount of payment guarantees with no liquidity obtained due to the discount and rediscount. In such a case, the liquidity cannot be obtained immediately by the traditional financing means. Because of the liquidity preference and the needs of improving the financial situation, the micro-credit company has the motivation of revitalizing the guarantee assets and enhancing its liquidity. Obviously, the securitization of

This research is supported by MOE (Ministry of Education in China) Project of Humanities and Social Sciences (No.17YJC630128), Fund Project of Natural Science Research in Colleges and Universities in Jiangsu Province (No.17KJB120005,No.17KJB120004), Fund Project of Philosophy and Social Science Research in Colleges and Universities in Jiangsu Province (No.2017SJB0233,N0.2017SJB0234). payment guarantees provides such a way for the micro-credit company. Via the true sale of assets of the securitization, the micro-credit company transfers payment guarantees to SPV to gain the liquidity. In sum, based on the purpose of studying of the theory of the asset securitization, improving of the microcredit company's liquidity, revitalizing the assets and reducing the risk, the research on securitization of payment guarantees has the profound significance for further research on the securitization and financial innovation.

However, there's little literature on payment guarantees of the micro-credit company and its securitization. There is only some qualitative research on the payment mechanism [6], legal issues [7] and the risk management issues of other guarantees [8]. Therefore, this paper researches the properties of payment guarantees of the micro-credit company and its securitization. Considering the important role of the pricing in the process of asset securitization, this paper focuses on the pricing of payment guarantee securitization. There is no doubt that the securitization of payment guarantees of the micro-credit company also belongs to the category of asset securitization, its pricing has in common with the pricing of the long-term assets securitization. Therefore, we study the pricing of the payment guarantees of the micro-credit company based on the existing pricing method of the long-term asset securitization. From the existing literature, we can find that the pricing methods of the long-term assets securitization mainly include: the prepayment model [9], the default probability model [10] and the options to adjust spreads (OAS) model [11]. Some scholars use artificial simulation method to price the securitization products [12]. Although the theoretical and empirical research of the prepayment model has made remarkable achievements, such kind of models relies too much on the historical data; it is difficult to forecast the results better once the corresponding environment changes. While the key point of the default model is to rely on historical data, the current macroeconomic environment and the debtor's financial situation. However, in practice, there is less data on the prepayment and the default of payment guarantees of the micro-credit company. In fact, the period of payment guarantees of the micro-credit company is shorter. For example, in Jiangsu province, the longest period of payment guarantees of the micro-credit company is 6 months. While in the short term, the possibility of the default is small, or the capital cushion can be set to cope with the possible default risk [4], or by buying insurance to transfer risk effectively. Therefore, we do not consider the condition of the default in the pricing process of this paper. Compared with the 
traditional statistical method, the artificial simulation method doesn't rely too much on historical data. However, this kind of method is still controversial because of the lack of theoretical basis. Compared with the aforementioned methods, the OAS model considers the interest rate fluctuation and the influence of implied options. The large amount of simulations of interest rate change path in OAS method provides reference for the research of this paper. This method is suitable for securitization pricing of medium and long term asset. However, payment guarantees of the micro-credit company are the short term assets, it cannot form a long-term stable cash flow during the process of securitization. Due the homogeneity of payment guarantees of the micro-credit company, inspired by research of [4], a portfolio of payment guarantees that can create a longterm stable cash flow is constructed.

In conclusion, the research on the guarantee securitization and its pricing is not sufficient at present, and the research is of great significance. Firstly, the design and the pricing of the guarantee securitization products can broaden the theory of asset securitization. The pricing of asset securitization products is mainly based on medium and long term credit assets with high risk at present. There is little research on the design and the pricing of short-term asset securitization with low risk, such as payment guarantees of the micro-credit company. Secondly, the guarantee securitization is easy to create the liquidity for guarantee assets, reduces the risk of guarantee assets and increases the profit of the micro-credit company. Considering this, the necessity, the process and the pricing of the payment guarantee securitization of the micro-credit company are respectively researched in this paper.

\section{Construction of the Asset PoOL}

The payment guarantee of the micro-credit company refers to the voucher issued by firms or personals opening accounts in the micro-credit company, and be accepted by the micro-credit company. The micro-credit company has the responsibility to pay certain amount to the payee or the person holding the voucher before the specified date according to the voucher.

\section{A. The necessity of securitization of payment guarantees}

(1) The micro-credit company meets the financing needs of SMEs and supports the three rural development, but its availability of external capital is limited [1].The micro-credit company is not allowed to absorb deposits from the public. In addition, the money which can be obtained from the bank also has a clear upper limit proportion in China. To address the micro-credit company's problem of financing difficulties, the scholars have given some ideas, such as raising its borrowing proportion in the bank[13] [14], transforming them into the village banks[15], or establishing institutions by national finance and other financial institutions that can injected cash flow into the micro-credit company [14][16]. However, compared to other companies' business, the risk of the microcredit company is bigger, leading to that banks are not willing to improve the loan ratio of the micro-credit company; Changing into the village banks means more stringent conditions. Moreover, the service objects of the micro-credit company are not only limited in rural areas, but also small and medium-sized enterprises located in the city. The last method needs the states' support and increases the financial burden. Due to this point, the last method reduce the momentum of business innovation and increases the probability of participation in high risk projects for the micro-credit company[4].Therefore, under such a background, obtaining liquidity via the financial innovation of their own products can be regarded as a kind of good choice. The securitization of payment guarantees of the micro-credit company can not only shed risky assets, but also make the micro-credit company obtain liquidity in advance, increasing the funds that can be lent and obtaining more profits.

(2) The discount and rediscount of payment guarantees of the micro-credit company occupy its own funds. The development of payment guarantees brings new income sources of income for the micro-credit company, such as issuing payment guarantees, discounting and rediscounting guarantees, transferring or reporting the loss of payment guarantees. At the meantime, discounting and rediscounting guarantees reduce the liquidity of the micro-credit company, leading to obtain lots of payment guarantees. Though the development of discount and rediscount business makes the micro-credit company obtain extra income, its liquidity becomes more insufficient. Besides, it is not easy to get liquidity from outside. It has great significance to research how to revitalize payment guarantees of the micro-credit company obtained from discount and rediscount business to making it gain liquidity in advance. The securitization of payment guarantees of the micro-credit company can be a good way.

In sum, the implementation of the securitization of payment guarantee of the micro-credit company is necessary.

\section{B. Cash flow design}

The duration of the payment guarantee of the micro-credit company is comparatively short, taking the payment guarantee of the micro-credit company in Jiangsu province as an example. The payment guarantee has six kinds of durations, namely one month, two months, three months, four months, five months and six months. Such short duration cannot offer sustainable cash flows. However, considering the homogeny of payment guarantees of the micro-credit company, an asset portfolio of payment guarantees with stable and sustainable cash flow can be constructed as shown in Fig.1.

For time-point 1, 2, 3, 4, 5, 6 (the time interval is one month), it can be constructed directly by several guarantees with corresponding ddurations to meet the cash flow of each period. For the time-points after time-point 6 , the cash flow cannot be constructed directly by guarantees with corresponding durations. In such a case, several kinds of payment guarantees should be gathered to form the corresponding cash flow. Taking time-point 7 for an example. The guarantee asset pool can be injected a guarantee with the duration of six months at time-point 1 or a guarantee with the duration of five months at time-point and so on. The timepoints after time-point 7 can be dealt with the same method, i.e. injecting payment guarantees with corresponding duration at the corresponding time point in advance. In order to acquire adequate guarantee asset to support expected cash flow, a capital buffer pool can be set up. The buffer capital equals the cash inflow of the asset pool in each period that designed in the 
guarantee securitization. If the cash inflow of the guarantee asset cannot support the cash inflows of each period, the buffer capital will make up the remaining cash flow. As long as the buffer capital is used, the cash will be injected into the buffer capital pool to make the buffer capital reach the initial value. This buffer capital also can be used to resist the loss of cash flow of the guarantee asset pool caused by default. Another solution is that several micro-credit companies jointly issue the payment guarantee backed securities and distribute the financing capital according to the amount of payment guarantees that they provide. In this way, more payment guarantees with different durations can be acquired and the corresponding risk can be shared.

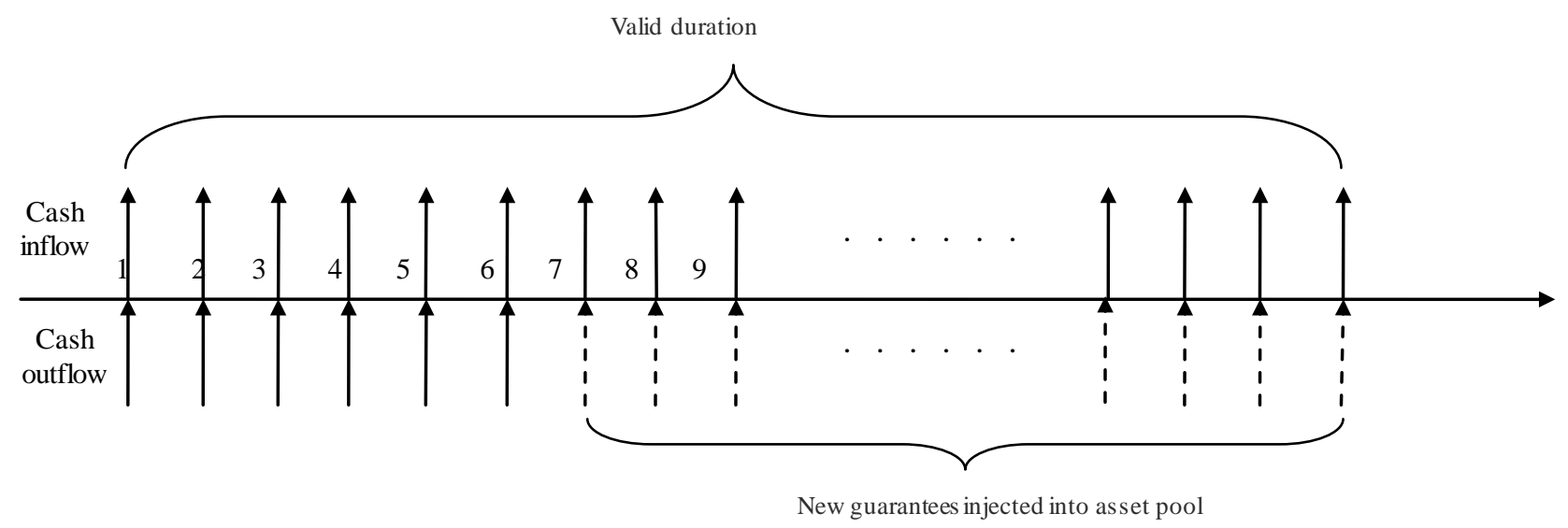

Fig. 1. Cash flow of asset pool

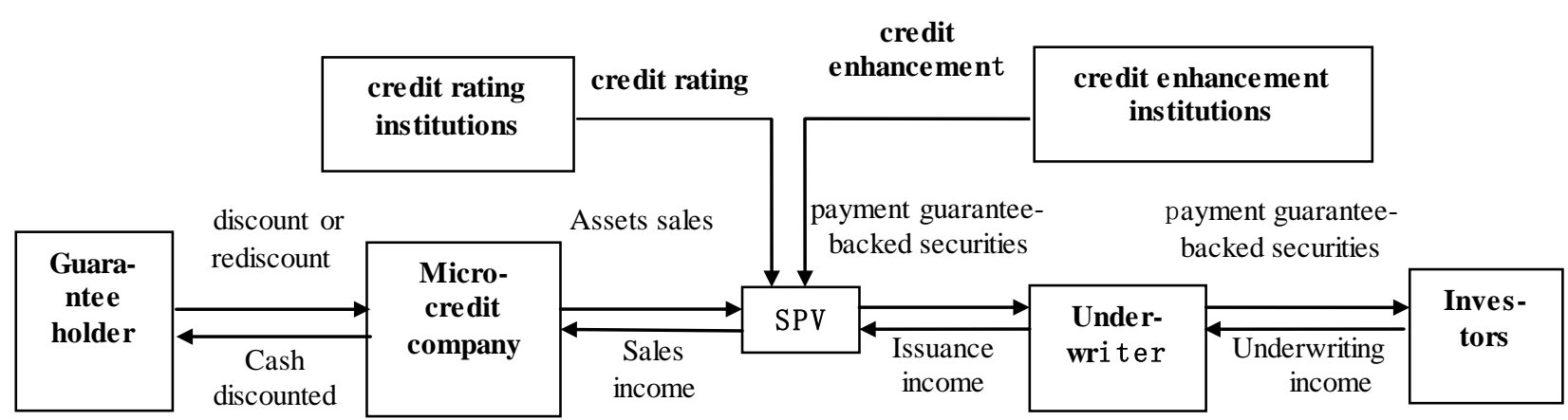

Fig. 2. Securitization Processes of Payment Guarantees

Cash flow of the securitized products constructed in this way can match the cash flow of the underlying asset in term very well. The advantage of this method is that it only needs one time issue and the corresponding cost is small. The cash inflows (matured recovery payment of guarantees) and outflows (interest payment of securities) of each period can be designed flexibly according to firms' financial needs to stabilize the cash flow of each period and reduce the pressure of interest payment in future. However, during the implementation process, (a) it's necessary to ensure enough expected cash inflows injected into the asset pool of each period. To achieve this goal, the capital buffer pool mentioned above needs to be set up. (b) Though the duration of the payment guarantee of the micro-credit company is short and the possibility of default is low, it is necessary to consider the unexpected risk. Therefore, insurance on payment guarantees in the capital pool can be used to reduce the default risk. (c) The department that is responsible for collecting payment guarantees should be set in order to obtain adequate payment guarantees with different kinds of durations to support the current cash inflow or outflow at each time-point. This department should construct the payment guarantee portfolio at each time-point 6 months in advance. The shortcoming of this method is that adequate payment guarantees are needed to satisfy the stable cash flow designed, which brings a great pressure to look for payment guarantees with kinds of durations needed, and the longest preparation time is 6 months. During the process of implementing this method, it's a good choice to establish a strict contractual relationship among numerous promising micro-credit companies to reduce the pressure of the acquisition of payment guarantees.

\section{Securitization Process of Payment Guarantee}

The securitization of payment guarantees of the microcredit company also belongs to the category of asset securitization. Therefore, once the payment guarantee asset pool is created, its securitization process is similar to the midlong term asset securitization process as shown in Fig. 2. The 
holder of payment guarantees seeks the liquidity from the micro-credit company by means of the discount or rediscount of payment guarantees. Due to the discount or rediscount, the micro-credit company holds lots of guarantees. For the need of liquidity preference and financing needs, the micro-credit company use payment guarantees with kinds of durations to form an asset portfolio which can produce steady cash flow and sell it to SPV at a certain price. SPV is responsible for designing the specific content of bonds based on this, employing credit enhancement institutions and credit rating institutions for credit enhancement and credit rating, and selecting the underwriters to issue the payment guaranteebacked securities of the micro-credit company to investors.

\section{Pricing of the Securitization of Payment GUARANTEES}

According to the above analysis, in the process of the securitization of payment guarantees of the micro-credit company, a capital buffer pool can be set up, in which the buffer capital equals the cash inflow or outflow of each period that designed in the guarantee securitization. When the buffer capital in this pool is used, the capital buffer pool will be replenished to the initial level in time. Firstly, if the cash flow of the guarantee asset portfolio of some period is less than the cash inflows of each period that designed in the guarantee securitization, the buffer capital can make up the remaining part. Secondly, the pool can also be used to resist the the loss resulted from defaults. In addition, due to short term of the payment guarantee of the micro-credit company, prepayment and default are not considered in pricing. Based on the aforementioned points, the pricing of the securitization of payment guarantees of the micro-credit company has two hypotheses: a. prepayment and default are not considered; b. guarantee asset pool has a fixed and stable cash inflow at each period. Under these two hypotheses, two key elements of pricing can be studied: change path of interest rate and cash flow.

\section{A. Dynamic interestrate model}

In this paper, a classical Vasicek model is selected to characterize changes in interest rates, which has mean reversion properties and has been used in term structure and pricing of derivatives by many scholars. The relative research shows that Vasicek model is suitable for Chinese financial markets to some extent [17]. In [18], the interbank lending rate for 30 days is used to estimate the parameters of Vasicek model, which can be expressed by the following equation:

$$
d r_{t}=0.022915\left(3.514655-r_{t}\right) d_{t}+0.752292 d z_{t}
$$

In this paper, the dynamic process in Eq.(1) is adopted.

\section{B. Pricing according to the cash flow of asset pool}

The parameters of the guarantee asset pool are set in this part: $w_{t}$ is the cash inflow of payment guarantee asset pool at time point; $r$ is the spot rate and $S$ is the service fees of financial institutions.

$N F_{t}$ is the cash inflows of asset pool at time point $t$ :

$$
N F_{t}=W_{t}-S
$$

Based on the Monte Carlo simulation, the value of guarantee-backed securities of the micro-credit company can be calculated under different change paths of interest rates:

$$
P_{i}=\frac{N F_{i 1}}{1+r_{i 1}+k}+\frac{N F_{i 2}}{\left(1+r_{i 2}+k\right)^{2}}+\cdots+\frac{N F_{i T}}{\left(1+r_{i T}+k\right)^{T}}
$$

\section{Simulation of the pricing of the securitization of payment guarantees}

Considering that there is no reference cases about the securitization of payment guarantees of the micro-credit company and we can not get the related parameters of guarantee assets. In order to give an example about the theoretical pricing of guarantee-backed securities of the micro-credit company, the simulation method is used. Due to that the service fees of financial intermediaries depend on the value of assets managed. During this simulation, assume that $N F_{t}$ is the same at each time point $t$ and the service fees of financial institutions are calculated according to the cash inflow at each time point $t$. The parameters are set as follows: $W_{t}=1$ million yuan, $s=0.3 \%, k=5 \%, T=10$ years. The above parameters are set for the purpose of simplifying the calculation process and without actual specific meaning. Matlab is used to simulate the above pricing process. The simulation is repeated 1000 times and 1000 prices of guarantee-backed securities can be obtained. The simulation results are reflected in Fig.3. The theoretical price of the payment guarantee-backed security equals 20.14 million yuan according to the mean value of 1000 times.

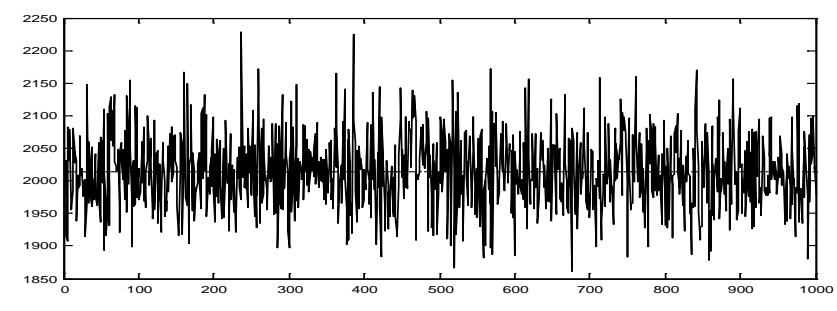

Fig. 3. Simulation graph of the price of guarantee-backed securities

\section{CONCLUSION}

The purpose of this research is to study the necessity of payment guarantee securitization of the micro-credit company, the construction of the payment guarantee asset pool, the process of payment guarantee securitization and the pricing of payment guarantee-backed securities of the micro-credit company, to make up the securitization theory of short-term underlying assets. This research has an important theoretical and academic value, and has an important practical and guiding significance for payment guarantee securitization of the micro-credit company as well. It can be foreseen that in the increasingly competitive financial environment, the implementation of payment guarantee securitization of the micro-credit company is beneficial to make the disposable capital to enter the financial markets. Concurrently, it will bring a new profitable growth point for the micro-credit company. 


\section{REFERENCES}

[1] Du Jun, Li Lianjun. The analysis on financial channels of the microcredit company in the perspective of financial-industrial integration [J]. Seeker, 2013 (10):35-37. (in Chinese)

[2] Liu Xuewen, Huang Weihao. The idea of building the small loan joint company[J]. Finance \& Economics, 2012 (6):26-34. (in Chinese)

[3] Qu Xiaogang, Luo jianzhao. Evaluation and impact factors of the commercial micro-credit companies' sustainable development [J]. Journal of Northwest A\&F University (Social Science Edition), 2013(3):98-103. (in Chinese)

[4] Hu Yinyi. The micro-credit company's financing "bottleneck" and the right of return on assets evaluation [J]. Reform,2013 (6):135-143. (in Chinese)

[5] Huang Xingpeng, Le Yun. The situations, problems and solutions of the micro-credit company's financing [J]. Zhejiang Finance,2013, (10):6366. (in Chinese)

[6] Li Yan. Research of bank' independent guarantees payment mechanism [J]. Social Science Research, 2008 (4):107-113. (in Chinese)

[7] Li Yan. The logical explanation of Independent guarantees' documentary and the choice of leg islation in our country [J]. Tribune of Political Science and Law, 2013, 31(4):129-135. (in Chinese)

[8] Hao Yan, Chu Beiping. The risk aversion of refund guarantee in ship building $[\mathrm{J}]$. Journal of Dongbei University of Finance and Economics,2012, (4):72-76. (in Chinese)

[9] Zhou T. Indifference valuation of mortgage-backed securities in the presence of prepayment risk [J]. Mathematical Finance, 2010, 20(3): 479-507.
[10] Kau J B, Keenan D C, Yildirim Y. Estimating default probabilities implicit in commercial mortgage backed securities (CMBS) [J]. The Journal of Real Estate Finance and Economics. 2009, 39(2): 107-117.

[11] Liu Z Y, Fan G, Lim K G. Extreme events and the copula pricing of commercial mortgage-backed securities [J].The Journal of Real Estate Finance and Economics, 2009, 38(3): 327-349.

[12] Zhang Wenqiang. A study on the risk pricing of accounts receivable backed securitization in entity enterprises [J]. Journal of Financial Research, 2009, (5):194-205. (in Chinese)

[13] Liu Guofang, Qi Limei. A study on development of rural small loan companies [J]. Economic Review, 2009(12):94-97. (in Chinese)

[14] Hu Qiulin, Sun Ruixia. A study on the problems and solutions of the development of western micro-credit companies[J].Yunnan Finance \& Economics University Journal of Economics \& Management(Social Science Edition),2010(3):60-62. (in Chinese)

[15] Gao Xiaoyan, Ren Lihua,Zhao Yin. Further research on sustainable operation of micro-loan company in China [J]. Modern Finance and Economics Journal of Tianjin University of Finance and Economics, 2011(3):15-22. (in Chinese)

[16] Xing Zaozhong. Research of sustainable development of microcredit companies [J]. Shanghai Finance, 2009 (11):5-11. (in Chinese)

[17] Xiao Wu. The research of management of interest risk based on embedded option in commercial bank [D]. Southwestern University of Finance and Economics, 2012. (in Chinese)

[18] Tang Z, He J, Sui X. Research on the pricing of guarantee-backed securities based on Monte Carlo simulation[C].Management Science \& Engineering (ICMSE), 2014 International Conference on. IEEE, 2014: 1274-1279. 